PROCEEDINGS OF THE

AMERICAN MATHEMATICAL SOCIETY

Volume 131 , Number 12, Pages 3839-3843

S 0002-9939(03)07116-8

Article electronically published on June 30, 2003

\title{
INDUCED LOCAL ACTIONS ON TAUT AND STEIN MANIFOLDS
}

\author{
ANDREA IANNUZZI
}

(Communicated by Mohan Ramachandran)

\begin{abstract}
Let $G=(\mathbb{R},+)$ act by biholomorphisms on a taut manifold $X$. We show that $X$ can be regarded as a $G$-invariant domain in a complex manifold $X^{*}$ on which the universal complexification $(\mathbb{C},+)$ of $G$ acts. If $X$ is also Stein, an analogous result holds for actions of a larger class of real Lie groups containing, e.g., abelian and certain nilpotent ones. In this case the question of Steinness of $X^{*}$ is discussed.
\end{abstract}

\section{INTRODUCTION}

Let $X$ be a complex manifold endowed with an action by biholomorphisms of a connected real Lie group $G$, i.e., $X$ is a complex $G$-manifold. If the Lie algebra of the universal complexification $G^{\mathbb{C}}$ of $G$ is the complexification of $\operatorname{Lie}(G)$, then one obtains an induced local $G^{\mathbb{C}}$-action by integrating the $\mathbb{C}$-linear extension of the infinitesimal generator associated to the $G$-action. In many cases this can be understood as the restriction of a global $G^{\mathbb{C}}$-action, that is, it is possible to realize $X$ as a $G$-invariant domain in a complex $G^{\mathbb{C}}$-manifold $X^{*}$ to which we will refer as a globalization of the local $G^{\mathbb{C}}$-action. For instance, by a result of P. Heinzner ([] $)$ if $X$ is Stein and $G$ compact, then there exists a Stein globalization $X^{*}$ with the following universal property: every holomorphic $G$-equivariant map on $X$ to a complex $G^{\mathbb{C}}$-manifold extends $G^{\mathbb{C}}$-equivariantly on $X^{*}$.

Furthermore, for $X$ Stein and $G$ with polar complexification $G^{\mathbb{C}}$ and cocompact discrete subgroup $\Gamma$ such that $G^{\mathbb{C}} / \Gamma$ is Stein, equivalent conditions for the existence of a Stein universal globalization are given in [CIT. These can be verified to hold in many concrete situations, however it seems not to be known whether in this setting a globalization always exists. Here we first consider $(\mathbb{R},+)$-actions on taut manifolds and we prove the following:

Let $X$ be a taut $\mathbb{R}$-manifold. Then there exists a universal globalization $X^{*}$ of the induced local $\mathbb{C}$-action.

Note that one cannot expect $X^{*}$ to be taut unless the $\mathbb{R}$-action on $X$ is trivial. If $X$ is also Stein, we show that a similar result holds for $G$ in the above-mentioned class of real Lie groups (Corollary 3). In this case it is natural to ask whether such

Received by the editors July 25, 2002.

2000 Mathematics Subject Classification. Primary 32M05, 32E10, 32 Q99.

Key words and phrases. Lie group actions, complexifications, taut and Stein manifolds.

This work was partially supported by the University of Bologna, funds for selected research topics. 
a universal globalization is also Stein. For $G=(\mathbb{R},+)$ it turns out that this is equivalent to a positive answer to the following open question:

Let $Y$ be a complex manifold and assume there exist lower semicontinuous functions $\alpha, \beta: Y \rightarrow \mathbb{R}$ such that $\Omega:=\{(\lambda, y) \in \mathbb{C} \times Y:-\beta(y)<\operatorname{Im} \lambda<\alpha(y)\}$ is Stein. Is $Y$ then Stein?

We conclude by pointing out particular cases where this holds true.

\section{EXISTENCE OF GLOBALIZATIONS}

For basic facts and results on local actions and their globalizations we refer to $[\mathrm{P}$ and more generally to [HI, $\S 11-3]$, from which most notations are inherited. However note that here all manifolds are assumed to be Hausdorff (cf. [HI, §3]).

Theorem 1. Let $X$ be a taut $\mathbb{R}$-manifold. Then there exists a universal globalization $X^{*}$ of the induced local $\mathbb{C}$-action.

Proof. Note that every leaf $\Sigma$ of Palais' foliation with respect to the induced local $\mathbb{C}$-action is a non-compact Riemann surface, since its projection $\left.p\right|_{\Sigma}: \Sigma \rightarrow \mathbb{C}$ is not constant. In particular $\Sigma$ is holomorphically separable and [HI, Corollary, p. 438] applies to show univalency of such a local action. Then by [HI, Theorem 2, p. 38] there exists a possibly non-Hausdorff universal globalization $X^{*}$. The result will follow by showing that $X^{*}$ is Hausdorff.

For this suppose that there exist elements $x_{1}$ and $x_{2}$ in $X^{*}$ which are not topologically separable. Since $X^{*}=\mathbb{C} \cdot X$ and $X$ is $\mathbb{R}$-invariant one may assume that $x_{1} \in X$ and $x_{2}=i t \cdot x_{0}$ with $x_{0} \in X$ and $t \in \mathbb{R}^{>0}$. Note that $X$ is Hausdorff, thus $x_{2} \notin X$ and consequently the local $\mathbb{C}$-orbit through $x_{0}$ has necessarily complex dimension one. Then one can choose a local slice $f: \mathbb{B}^{n-1}(1) \rightarrow X$ transversal to $\mathbb{C} \cdot x_{0}$ with $f(0)=x_{0}$ and a neighborhood $U \subset \mathbb{C}$ of 0 such that $\varphi: U \times \mathbb{B}^{n-1}(1) \rightarrow X$ defined by $\varphi(z, s):=z \cdot f(s)$ is a chart of $X$. Here $n$ is the complex dimension of $X$ and $\mathbb{B}^{n-1}(r):=\left\{s \in \mathbb{C}^{n-1}:|s|<r\right\}$ for all $r>0$. Let us call such a chart an adapted chart of $X$ in $x_{0}$.

Now it $\cdot \varphi\left(r U \times \mathbb{B}^{n-1}(r)\right)$ are open neighborhoods of $x_{2}$ for all $0<r<1$ and we are assuming that $x_{1}$ and $x_{2}$ are not separable. Therefore there exists a sequence $\left(z_{j}, s_{j}\right)$ convergent to $(0,0)$ in $U \times \mathbb{B}^{n-1}(1)$ such that $X \ni i t \cdot \varphi\left(z_{j}, s_{j}\right) \rightarrow x_{1}$. Thus for $y_{j}:=\varphi\left(z_{j}, s_{j}\right)$ one has $X \ni y_{j} \rightarrow x_{0}$ and $X \ni i t \cdot y_{j} \rightarrow x_{1}$. Now recall that $X$ is orbit-connected (cf. [CIT, Lemma 1.6]) and $\mathbb{R}$-invariant in $X^{*}$. Then by considering an adapted chart of $X$ in $x_{1}$ one checks that there exists $\epsilon>0$ such that $S:=\{z \in \mathbb{C}:-\epsilon<\operatorname{Im} z<t+\epsilon\} \subset \Omega\left(y_{j}\right)$ for all $j>0$, where by definition $\Omega(x):=\{z \in \mathbb{C}: z \cdot x \in X\}$ for all $x \in X$.

Define a sequence of holomorphic functions $h_{j}: S \rightarrow X$ by $h_{j}(z):=z \cdot y_{j}$, let $a_{0}, b_{0} \in \mathbb{R}^{>0}$ be given by $\Omega\left(x_{0}\right)=\left\{z \in \mathbb{C}:-b_{0}<\operatorname{Im} z<a_{0}\right\}$ and note that $i t \cdot x_{0} \notin X$, hence $a_{0} \leq t$. Moreover $h_{j}(0) \rightarrow x_{0}$ while $i a_{0} \cdot x_{0} \notin X$ and $i s \cdot x_{0} \in X$, for $s$ smaller than $a_{0}$ and close to it, imply that $h_{j}\left(a_{0}\right) \rightarrow \infty$. Since $X$ is taut, this gives a contradiction and concludes the proof.

Remark 2. Since $X$ is $\mathbb{R}$-invariant and orbit-connected in $X^{*}$, there exist lower semicontinuous positive functions $a, b: X \rightarrow \mathbb{R}^{>0}$ such that

$$
\Omega(x)=\{z \in \mathbb{C}:-b(x)<\operatorname{Im} z<a(x)\}
$$


for all $x$ in $X$, where $\Omega(x):=\{z \in \mathbb{C}: z \cdot x \in X\}$. An analogous argument as in the above proof applies to show that on a taut manifold, $a$ and $b$ are continuous (if $X$ is Stein one knows that $-a$ and $-b$ are plurisubharmonic [F]).

Let $G$ be a real Lie group with polar complexification $G^{\mathbb{C}}$, i.e., the $G$-equivariant map $G \times \mathfrak{g} \rightarrow G^{\mathbb{C}}$ given by $(g, \xi) \rightarrow g \exp i \xi$ is a real analytic diffeomorphism. Furthermore assume that $G$ admits a discrete cocompact subgroup $\Gamma$ such that $G^{\mathbb{C}} / \Gamma$ is Stein. For instance all abelian and compact real Lie groups are of this kind or more generally products of the form $K \times N$, with $K$ compact and $N$ simply connected and nilpotent with rational structure constants (see [Ma, $[\mathrm{GH}]$ ). Since $G^{\mathbb{C}}$ is polar, the Lie algebra of $G^{\mathbb{C}}$ is the complexification of $\mathfrak{g}$, the Lie algebra of $G$. As a consequence if $G$ acts on a complex manifold one obtains a holomorphic local action of the complexification $G^{\mathbb{C}}$ by integrating the holomorphic vector fields given by the $G$-action. For $G$ as above one has

Corollary 3. Let $X$ be a taut and Stein $G$-manifold. Then there exists a universal globalization $X^{*}$ of the induced local $G^{\mathbb{C}}$-action.

Proof. For $\eta \in \mathfrak{g}$, consider the $\mathbb{R}$-action on $X$ defined by $t \cdot x:=(\exp t \eta) \cdot x$ and denote by $X_{\eta}^{*}$ the universal globalization of the induced local $\mathbb{C}$-action given by the above theorem. Then the corollary is a consequence of [CIT, Corollary 3.7].

For an action of a compact Lie group $G$ on a Stein manifold the universal globalization $X^{*}$ is automatically Stein $([\mathrm{H}])$. It would be interesting to know whether this remains true in the case where $G$ is not compact and $X^{*}$ exists. For $G=\mathbb{R}$ one has

Proposition 4. The following statements are equivalent:

i) Let $X$ be a Stein $\mathbb{R}$-manifold with universal globalization $X^{*}$. Then $X^{*}$ is Stein.

ii) Let $Y$ be a complex manifold and assume there exist lower semicontinuous functions $\alpha, \beta: Y \rightarrow \mathbb{R}$ such that $\Omega:=\{(\lambda, y) \in \mathbb{C} \times Y:-\beta(y)<$ $\operatorname{Im} \lambda<\alpha(y)\}$ is Stein. Then $Y$ is Stein.

Proof. Let $\Omega$ be as in ii) and consider the $\mathbb{R}$-action by left multiplication on the first component of $\mathbb{C} \times Y$. Then [CIT, Lemma 1.5] applies to show that $\mathbb{C} \times Y$ is the universal globalization of $\Omega$. Thus if i) holds, then $\mathbb{C} \times Y$ is Stein and consequently so is $Y$, implying ii).

Conversely for $X$ as in i) let $\mathbb{R}$ act diagonally on $\mathbb{C} \times X$ and by left multiplication on the first component of $\mathbb{C} \times X^{*}$. Then the map $f: \mathbb{C} \times X \rightarrow \mathbb{C} \times X^{*}$ given by $(\lambda, x) \rightarrow\left(\lambda, \lambda^{-1} \cdot x\right)$ is easily checked to be an $\mathbb{R}$-equivariant open embedding. In particular $f(\mathbb{C} \times X)$ is a Stein $\mathbb{R}$-invariant subdomain of $\mathbb{C} \times X^{*}$.

Now let $a, b: X \rightarrow \mathbb{R}^{>0}$ be as in Remark 2, fix $y \in X^{*}$ and choose $x \in X$ and $t \in \mathbb{R}$ such that $y=i t \cdot x$. One has that

$$
(\lambda, y)=\left(\lambda, \lambda^{-1} \cdot((\lambda+i t) \cdot x)\right)
$$

belongs to $f(\mathbb{C} \times X)$ if and only if $(\lambda+i t) \cdot x \in X$, i.e., $-b(x)-t<\operatorname{Im} \lambda<a(x)-t$. By defining $\alpha(y)=a(x)-t$ and $\beta(y)=b(x)+t$ (which is easily verified not to depend on the choice of $x$ and $t$ ) for all $y \in X^{*}$ one has

$$
f(\mathbb{C} \times X)=\left\{(\lambda, y) \in \mathbb{C} \times X^{*}:-\beta(y)<\operatorname{Im} \lambda<\alpha(y)\right\}
$$


and statement i) follows from ii) by letting $\Omega=f(\mathbb{C} \times X)$ in $\mathbb{C} \times X^{*}$, which concludes the proof.

Remark 5. In the following cases it is easy to check that statement ii) holds:

1) $Y$ is holomorphically convex.

For this, first note that for any open Stein neighborhood $U$ in $Y$ the restrictions of $-\alpha$ and $-\beta$ to $U$ define the Stein domain $\Omega \cap(\mathbb{C} \times U)$ in $\mathbb{C} \times U$. It follows that $-\alpha$ and $-\beta$ are plurisubharmonic (see, e.g., $[\mathbf{V}$ ).

Now recall that each fiber $F$ of the Remmert reduction of $Y$ (cf. [GR, p. 221]) is a connected compact subspace. In particular $\alpha$ and $\beta$ are constant on $F$, thus $F \cong\{z\} \times F \subset \Omega$ for any fixed $z$ in $\mathbb{C}$ with $-\left.\beta\right|_{F}<\operatorname{Im} z<\left.\alpha\right|_{F}$ and consequently $F$ is holomorphically separable. By compactness and connectness it follows that $F$ consists of a single point, hence $Y$ is Stein.

2) $Y$ is a domain in a Stein manifold $\hat{Y}$.

Here $\Omega$ can be regarded as an open Stein $\mathbb{R}$-invariant subdomain of $\mathbb{C} \times \hat{Y}$, where $\mathbb{R}$ acts by left multiplication on the first component. Since $\mathbb{C} \times \hat{Y}$ is Stein, then $\Omega$ is locally Stein ( $[\mathrm{DG}])$.

Moreover the quotient map $\mathbb{C} \times \hat{Y} \rightarrow(\mathbb{C} \times \hat{Y}) / \mathbb{Z}$ is locally biholomorphic, therefore $\Omega / \mathbb{Z}$ is locally Stein in $(\mathbb{C} \times \hat{Y}) / \mathbb{Z} \cong \mathbb{C}^{*} \times \hat{Y}$, which is Stein, and consequently so is $\Omega / \mathbb{Z}$. Finally $Y$ is easily checked to be biholomorphic to the categorical quotient of $\Omega / \mathbb{Z}$ with respect to the natural induced $S^{1}$-action, thus it is Stein $([\mathrm{H}$. $\S 6.5])$.

Remark 6. As already noted in the proof of Theorem 1 , a complex $\mathbb{R}$-manifold admits a universal globalization $X^{*}$ which is possibly non-Hausdorff. Note that the same argument used to prove Proposition 4 applies to show the analogous result in the case where $X^{*}$ and $Y$ are assumed to be in the category of possibly non-

Hausdorff complex manifolds.

\section{REFERENCES}

[A] M. Abate, Iteration Theory of Holomorphic Maps on Taut Manifolds, Research and Lecture Notes in Mathematics. Complex Analysis and Geometry, Mediterranean Press, Cosenza, 1989. MR 92i:32032

[CIT] E. Casadio Tarabusi, A. Iannuzzi, and S. Trapani, Globalizations, fiber bundles and envelopes of holomorphy, Math. Z. 233 (2000), 535-551. MR 2001f:32035

[DG] F. Docquier and H. Grauert, Leisches Problem und Rungescher Satz für Teilgebiete Steinscher Mannigfaltigkeiten, Math. Ann. 140 (1960), 94-123. MR 26:6435

$[\mathrm{F}] \quad$ F. Forstnerič, Actions of $(\mathbb{R},+)$ and $(\mathbb{C},+)$ on complex manifolds, Math. Z. 223 (1996), 123-153. MR 97i:32041

[GH] B. Gilligan and A. T. Huckleberry, On non-compact complex nil-manifolds, Math. Ann. 238 (1978), 39-49. MR 80a:32021

[GR] H. Grauert and R. Remmert, Coherent Analytic Sheaves, Grundlehren der Mathematischen Wissenschaften, Vol. 265, Springer-Verlag, Berlin, Heidelberg, New York, Tokyo, 1984. MR 86a:32001

[H] P. Heinzner, Geometric invariant theory on Stein spaces, Math. Ann. 289 (1991), 631-662. MR 92j:32116

[HI] P. Heinzner and A. Iannuzzi, Integration of local actions on holomorphic fiber spaces, Nagoya Math. J. 146 (1997), 31-53. MR 98k:32047.

[I] A. Iannuzzi, Characterizations of G-tube domains, Manuscripta Math. 98 (1999), 425-445. MR 2000e:32034

[Ma] A. I. Mal'čev, On a class of homogeneous spaces, Amer. Math. Soc. Translation, vol. 39, Amer. Math. Soc., Providence, RI, 1951. MR 12:589e 
[P] R. S. Palais, A global formulation of the Lie theory of transformation groups, Mem. Amer. Math. Soc., vol. 22, Amer. Math. Soc., Providence, RI, 1957. MR 22:12162

[V] V. S. Vladimirov, Les fonctions de plusieurs variables complexes et leur application à la théorie quantique des champs, Dunod, Paris, 1967. MR 36:1692

Dipartimento di Matematica, Università di Bologna, Piazza di Porta S. Donato 5, I-40126 BolOGNA, ITALY

E-mail address: iannuzzi@dm.unibo.it 